\title{
Dexametasona transtimpánica para el tratamiento de la enfermedad de Ménière de difícil manejo
}

\section{Transtympanic dexamethasone injections for the management of refracatory Ménière's disease}

\author{
Francisco García-Huidobro N. ${ }^{1}$, Andrés Rosenbaum F. ${ }^{1}$, Sofia Waissbluth A. ${ }^{1}$, \\ Karina Aracena C. ${ }^{1}$, Héctor Riveros $M .{ }^{1}$
}

\section{Resumen}

Introducción: La enfermedad de Ménière se caracteriza por crisis recurrentes de vértigo, asociado a hipoacusia y tinnitus, que pueden ser incapacitantes, y provocar estrés y ansiedad. Objetivos: Evaluar el efecto de la dexametasona transtimpánica sobre la audición y vértigo en pacientes con enfermedad de Ménière de difícil manejo. Material y Método: Se reclutó a pacientes con enfermedad de Ménière de difícil manejo médico quienes fueron tratados con tres inyecciones transtimpánicas de dexametasona $(4 \mathrm{mg} / \mathrm{ml})$. Se realizó audiometría, prueba calórica y dos encuestas de discapacidad (DHI y UCLA-DQ) al inicio, tres, y diez semanas posintervención, además de una entrevista a los seis meses. Resultados: Doce pacientes completaron el estudio. Se observó un aumento del promedio de umbrales auditivos hasta $11,25 \mathrm{~dB}$, principalmente entre 125 y $3000 \mathrm{~Hz}$ a las tres semanas, y una reducción de éstos hasta $7,5 \mathrm{~dB}$ a las diez semanas respecto de los iniciales, principalmente entre 125 y $2000 \mathrm{~Hz}$ (no estadísticamente significativo). Todos los pacientes presentaron hipoexcitabilidad vestibular. Se observó una mejoría significativa en el puntaje de la encuesta UCLA-DQ posintervención, manteniéndose durante todo el seguimiento. No se encontraron diferencias estadísticamente significativas en el puntaje global ni subcomponentes de la encuesta DHI. Siete de ocho pacientes reportaron que volverían a utilizar las inyecciones. Conclusión: Los corticoides transtimpánicos pueden ayudar a disminuir la discapacidad e impacto en la calidad de vida de los pacientes con enfermedad de Ménière de difícil manejo a mediano y largo plazo.

Palabras clave: Enfermedad de Ménière, hidrops, vértigo, corticoides, transtimpánico.

\begin{abstract}
Introduction: Ménière's disease is characterized by recurrent episodes of vertigo, sensorineural hearing loss and aural fullness, which can be incapacitating and cause stress and anxiety. Aim: To assess the effect of transtympanic steroid injections on hearing and vertigo for patients suffering from intractable Ménière's disease. Material and Method: Patients with intractable Ménière's disease were recruited and treated with three transtympanic steroid injections (dexamethasone $4 \mathrm{mg} / \mathrm{ml}$ ). Audiometric and vestibular assessment, and two dizziness questionnaires (DHI, UCLA-DQ) were performed upon recruitment, three weeks, and ten weeks following the transtympanic treatment. A brief auto-perception survey was applied at 6 months follow-up. Results: Twelve patients completed the study. An increase in the mean auditory threshold was observed up to $11.25 \mathrm{~dB}$, mainly between 125 and $3000 \mathrm{~Hz}$ at three weeks, and a reduction of these up to $7.5 \mathrm{~dB}$ at ten weeks with respect to the initial ones, mainly between 125 and $2000 \mathrm{~Hz}$ (not statistically significant). All patients presented vestibular dysfunction on caloric testing. A significant improvement in the UCLA-DQ survey score was observed, remaining throughout the follow-up. No statistically significant differences were found in the overall score or subcomponents of the DHI. Seven out of eight patients reported that they would undergo the injections again. Conclusion: Transtympanic corticosteroids can help to reduce disability and impact on the quality of life of patients with Ménière's disease that is difficult to manage in the medium and long term.
\end{abstract}

Keywords: Ménière`s disease, hydrops, vertigo, corticosteroids, transtympanic.
${ }^{1}$ Departamento de Otorrinolaringología, Red de Salud UC Christus, Pontificia Universidad Católica de Chile. Santiago, Chile.

Los autores declaran no tener conflictos de interés.

Recibido el 15 de junio de 2020. Aceptado el 11 de septiembre de 2020 .

\section{Correspondencia:}

Sofia Waissbluth A. Departamento de Otorrinolaringología Pontificia Universidad Católica de Chile.

Marcoleta 352.

Santiago, Chile. Email: sofia.waissbluth@gmail. com 


\section{Introducción}

La enfermedad de Ménière es una condición compleja y multifactorial que clásicamente se caracteriza por la presencia de episodios recurrentes de vértigo espontáneo, hipoacusia, tinnitus y plenitud aural ${ }^{1,2}$. Es una patología crónica con un gran impacto en la calidad de vida de los pacientes. El compromiso puede llegar a ser bilateral, y la mayoría de los pacientes presentarán hipoacusia progresiva. $\mathrm{Si}$ bien se han podido asociar, mediante estudios histopatológicos los síntomas de la patología con la presencia de hidrops endolinfático, el mecanismo por el cual este se produce aún es desconocido ${ }^{1}$. Esto nos lleva al modelo de pensamiento actual donde el hidrops es considerado un epifenómeno asociado a múltiples trastornos del oído interno, siendo los alérgenos, factores genéticos y fenómenos autoinmunes las tres grandes hipótesis en la actualidad $^{3}$.

Dada la evolución heterogénea y variada de la enfermedad, la cual puede manifestarse como episodios de vértigo e hipoacusia fluctuante, actualmente no existe un algoritmo terapéutico estandarizado para su manejo ${ }^{3}$. Además, no existe claridad sobre los subtipos de la enfermedad que también podrían afectar los resultados de los tratamientos evaluados en trabajos científicos. Sin embargo, la gran mayoría de las publicaciones concuerda en comenzar con un manejo más bien conservador ${ }^{4,5}$. Este se caracteriza por el tratamiento de las crisis y la instauración de un tratamiento preventivo. Para este último, se ha sugerido iniciar un régimen hiposódico, usar betahistina, diuréticos o corticoides orales ${ }^{4-7}$. Además, se sugiere tratar factores asociados como por ejemplo, alergia, migraña, entre otros ${ }^{1,4}$. Con la instauración de estas medidas, es posible lograr un control de los síntomas en hasta un $80 \%$ de los $\operatorname{casos}^{5,8}$. En los pacientes de difícil manejo, es decir, aquellos en los cuales no es posible controlar la enfermedad con las medidas anteriormente expuestas, se puede iniciar un tratamiento con corticoides transtimpánicos. Otras alternativas para cuadros refractarios incluyen el uso de gentamicina transtimpánica y cirugía del saco endolinfático y cirugías mayores para casos rebeldes (i.e. laberintectomía, sección del nervio vestibular $)^{5,9,10}$.
Aun cuando existe evidencia que avala el uso de corticoides transtimpánicos en relación al tratamiento de otras patologías del oído interno ${ }^{11}$, en la enfermedad de Ménière la evidencia es variada, existiendo estudios que muestran algún efecto en el control y duración de las crisis de vértigo ${ }^{10}$. Recientemente, la Academia Americana de Otorrinolaringología y Cirugía de Cabeza y Cuello (AAO-HNS) publicó una guía de práctica clínica para la enfermedad de Ménière la cual propone, como opción, el uso de corticoides transtimpánicos en pacientes con enfermedad activa que no responden a terapias no invasivas ${ }^{12}$. Esta opción terapéutica es considerada en el manejo de la enfermedad de Ménière debido a la asociación de mecanismos autoinmunes e inflamatorios en la fisiopatología de esta enfermedad, los cuales han sido descritos por múltiples autores $^{1,3,13}$. Además, se ha demostrado que los corticoides mejoran la circulación a nivel del laberinto y que, consecuentemente, pueden mejorar la función del oído interno mediante el transporte de iones, mecanismos que influyen en la homeostasis de los fluidos cocleares ${ }^{3}$. Aun así, describen que existe una gran variabilidad en los protocolos; cantidad de inyecciones, tiempo entre inyecciones y concentraciones usadas. Lo más frecuentemente descrito es el uso de dexametasona o metilprednisolona ${ }^{12}$.

\section{Objetivo}

Sobre la base de lo anterior, el presente trabajo pretende presentar el protocolo que usamos habitualmente, y nuestra experiencia con el uso de dexametasona transtimpánica sobre el nivel de audición y grado de vértigo en los pacientes con enfermedad de Ménière de difícil manejo médico. Además, como objetivo secundario se buscó evaluar el impacto, el grado de discapacidad y la autopercepción de los pacientes ante su condición utilizando encuestas validadas en nuestro país (UCLADQ y DHI) y un cuestionario.

\section{Material y Método}

\section{Reclutamiento de los pacientes}

El presente estudio fue aprobado por el comité de ética de la Pontificia Universidad 
Católica de Chile, obteniendo consentimiento informado de todos los participantes. Se realizó un estudio de cohorte prospectivo observacional, en el cual se invitó a participar a todos los pacientes con diagnóstico de enfermedad de Ménière definitiva, definida según los criterios de la AAO-HNS del año 1995, de difícil manejo médico y con indicación de tratamiento con inyecciones de corticoides transtimpánicas ${ }^{14}$. Aunque existen criterios diagnósticos más recientes por parte de la Sociedad de Bárány, este proyecto fue elaborado el mismo año de la publicación, por lo cual se mantuvieron los criterios antes mencionados para el reclutamiento de los pacientes ${ }^{15}$. Todos los pacientes reclutados fueron atendidos en el policlínico de otoneurología del Centro de Especialidades Médicas de la Pontificia Universidad Católica de Chile, y debían presentar cuadros vertiginosos de difícil manejo. Esto fue definido como la persistencia de crisis de vértigo afectando la calidad de vida a pesar de un tratamiento médico bien indicado.

A todos los pacientes con estas características y a quienes se le ofreció tratamiento con corticoides transtimpánicos, se les invitó a participar y permitir acceso a los datos de una audiometría tonal y prueba calórica realizada con videonistagmografía previo al tratamiento, a las tres semanas y a las diez semanas posterior al inicio del tratamiento. Se les solicitó, además, contestar encuestas en relación al impacto del vértigo en su calidad de vida.

Los pacientes con los siguientes criterios fueron excluidos: menores de edad, enfermedad de Ménière bien controlada, antecedentes de alergia, anafilaxia o urticaria con el uso de dexametasona, embarazadas o con lactancia materna, pacientes con membrana timpánica alterada (otitis media crónica, perforación timpánica, retracciones timpánicas, otitis media aguda, miringitis, abundante timpanosclerosis), pacientes con un conducto auditivo externo que no permite visualización adecuada de membrana timpánica (i.e. exostosis del canal importante, tumores del canal, estenosis adquirida del canal, procidencia de pared anterior del canal importante), pacientes con diagnóstico de vértigo concomitante que no permite evaluar el efecto de las inyecciones de corticoides.

\section{Audiometría tonal}

Este examen se realizó con un audiómetro marca GSI ${ }^{\mathrm{TM}}$. El paciente debía estar sentado en una cabina insonorizada, donde se midieron los umbrales auditivos para las siguientes frecuencias: 128, 256, 512, 1024, 2048, 3000, 4096, 6000 y 8196 Hz. Se calculó el promedio tonal puro con las frecuencias de 500 a 4096 Hz. Luego se realizó discriminación de la palabra con bisílabos.

\section{Prueba calórica}

Para este examen, se colocó al paciente en decúbito supino con la cabeza elevada en $30^{\circ}$ grados y con el videonistagmógrafo instalado. Se estimula de forma unilateral con un estímulo con aire a $24^{\circ}$ y luego $48^{\circ}$ Celsius. Se registraron los siguientes hallazgos: vértigo, náuseas o vómitos, nistagmo (amplitud, dirección), y los valores de velocidad de la componente lenta (VCL). Un valor de VCL menor a $6 \%$ (rango de normalidad: $6-75 \%$ s) y un porcentaje de asimetría $\geq 25 \%$ se consideró como un examen alterado $^{16,17}$.

\section{Inyecciones transtimpánicas}

Se colocaron tres inyecciones de dexametasona transtimpánica en el oído afectado, con una concentración de $4 \mathrm{mg} / \mathrm{ml}$, en un volumen variable entre $0,3-0,8 \mathrm{ml}$, y dentro de 10 días. Para las inyecciones, se realiza un aseo del conducto auditivo externo bajo visión microscópica si fuera necesario, y se confirma indemnidad de la membrana timpánica. Luego, se coloca dentro de conducto lidocaína al 10\% durante 10 minutos para anestesiar la zona. Se aspira la lidocaína y se realiza aseo del conducto auditivo externo con suero fisiológico asegurando ausencia de líquido en el conducto. Luego, con el uso de un catéter de punción lumbar con aguja $27 \mathrm{G}$, se realiza una punción mediante técnica estéril en el cuadrante anteroinferior de la membrana timpánica y se procede a instilar entre 0,3-0,8 $\mathrm{ml}$ de dexametasona, variable según características del paciente. Se le solicita al paciente mantenerse en posición decúbito lateral contralateral y evitar deglutir durante 10 minutos.

\section{Encuestas}

Se utilizó la encuesta UCLA dizziness questionnaire (UCLA-DQ), para la evaluación del impacto del vértigo en el individuo, y el $D i$ - 
zziness Handicap Index (DHI) para evaluar el grado de discapacidad percibida por el paciente producto del vértigo. La encuesta UCLA-DQ contiene 5 preguntas con un puntaje máximo de 25 puntos, que permite evaluar la frecuencia de las crisis, la intensidad, el impacto que esto tiene en actividades de la vida diaria, calidad de vida y grado de miedo del paciente de presentar una nueva crisis. A mayor puntaje mayor impacto causa el vértigo en el individuo. Además, es posible evaluar la severidad de las crisis de vértigo al multiplicar los primeros dos ítems de la encuesta los cuales corresponden a frecuencia e intensidad de las crisis, respectivamente.

Por otro lado, el DHI comprende 25 preguntas con un puntaje máximo de 100 puntos, que abarcan problemas emocionales, físicos y funcionales con opciones de respuesta, siempre, a veces o nunca. A mayor puntaje, mayor grado de discapacidad. Además, es posible evaluar cada uno de los aspectos que componen la encuesta en forma individual, con puntajes máximos de 36 puntos para los problemas emocionales y funcional, y 28 puntos para los problemas físicos ${ }^{18}$. A mayor puntaje mayor alteración de la subcategoría. Ambas encuestas se encuentran validadas en el idioma español y la encuesta DHI tiene una homologación lingüística reciente en nuestro país ${ }^{18,19}$.

Se realizó un cuestionario breve que constaba de tres preguntas, el cual tenía como objetivo evaluar la autopercepción del paciente frente a su condición, a los seis meses después de haber recibido el tratamiento con las inyecciones de corticoides. Las preguntas fueron:

1. ¿Durante los últimos seis meses, ha tenido alguna crisis de vértigo?

a) Si tuvo crisis, estas son de mayor, igual o menor intensidad (los síntomas).

b) Si tuvo crisis, estas son de mayor, igual o menor duración.

2. ¿Se volvería a colocar inyecciones de corticoides transtimpánicas por sus crisis de Ménière?

3. ¿Siente menos ansiedad en relación a que le ocurra una nueva crisis por haberse colocado las inyecciones?

\section{Análisis estadístico}

Se utilizó promedio-desviación estándar (DE) para estadística descriptiva. Se utilizaron test no paramétricos (rangos signados de
Wilcoxon) para comparación de promedios. Se consideró estadísticamente significativo un valor $P<0,05$.

\section{Resultados}

\section{Demográficos}

Se reclutaron diecisiete pacientes a los que se les administraron tres dosis de corticoides transtimpánicos. De ellos, solo doce completaron al menos el control inicial, el de las tres semanas y la encuesta al término del estudio. Siete pacientes completaron el control de las diez semanas. Cinco pacientes no se presentaron a los controles posteriores a la intervención, por lo que fueron excluidos del estudio. De los doce pacientes incluidos, seis $(6 / 12 ; 50 \%)$ eran de sexo masculino. La mediana de edad fue de 54 años, con un rango entre 38 y 72 años.

Todos los pacientes recibieron como tratamiento inicial, régimen hiposódico y betahistina de $24 \mathrm{mg}$ cada 12 horas por al menos dos meses (media: 2 meses, rango 2-12 meses) sin mejoría clínica previo al uso de corticoides transtimpánicos. Seis pacientes (50\%) se presentaron inicialmente con enfermedad de Ménière del oído derecho, cinco del oído izquierdo (42\%) $\mathrm{y}$ un paciente con enfermedad de Ménière bilateral $(8 \%)$. Además, se observó que once de los doce pacientes $(91,67 \%)$ presentaron tinnitus al inicio de la terapia. Cuatro de los doce pacientes incluidos (33\%) habían recibido un ciclo de corticoides transtimpánicos previo a la presente intervención, con una mediana de tiempo de 30 meses respecto al ciclo anterior (rango 7-96 meses).

\section{Audiometría tonal}

Se obtuvieron los umbrales auditivos al inicio del estudio, a las tres, y diez semanas posintervención, las cuales se muestran en la Tabla 1.

Se observó un aumento en los umbrales promedio de hasta $11,25 \mathrm{~dB}$ entre el control inicial y el control a las tres semanas que se concentran principalmente entre las frecuencias 125 y 3000 Hz. Sin embargo, en el control de las diez semanas, se observa una reducción en los umbrales promedio de hasta $7,5 \mathrm{~dB}$ al compararlo con el control inicial, entre las frecuencias 125 y $2000 \mathrm{~Hz}$. Por otro lado, entre los 3000 y $8000 \mathrm{~Hz}$, se observa un aumento de 


\begin{tabular}{|c|c|c|c|c|c|c|c|c|c|}
\hline & $125 \mathrm{~Hz}$ & $250 \mathrm{~Hz}$ & $500 \mathrm{~Hz}$ & $1000 \mathrm{~Hz}$ & $2000 \mathrm{~Hz}$ & $3000 \mathrm{~Hz}$ & $4000 \mathrm{~Hz}$ & $6000 \mathrm{~Hz}$ & $8000 \mathrm{~Hz}$ \\
\hline Inicial & $\begin{array}{c}57,5 \\
(13,9)\end{array}$ & $\begin{array}{c}59,58 \\
(13,73)\end{array}$ & $\begin{array}{l}57,08 \\
(18,4)\end{array}$ & $\begin{array}{c}54,17 \\
(16,76)\end{array}$ & $\begin{array}{c}48,33 \\
(19,23)\end{array}$ & $\begin{array}{c}48,75 \\
(17,96)\end{array}$ & $\begin{array}{c}52,5 \\
(19,24)\end{array}$ & $\begin{array}{c}59,58 \\
(22,81)\end{array}$ & $\begin{array}{c}62,08 \\
(20,50)\end{array}$ \\
\hline 3 semanas & $\begin{array}{c}62,08 \\
(14,84)\end{array}$ & $\begin{array}{l}65,83 \\
(16,9)\end{array}$ & $\begin{array}{c}66,67 \\
(18,38)\end{array}$ & $\begin{array}{c}65,42 \\
(19,59)\end{array}$ & $\begin{array}{c}55,83 \\
(21,76)\end{array}$ & $\begin{array}{c}53,75 \\
(21,65)\end{array}$ & $\begin{array}{c}55 \\
(23,93)\end{array}$ & $\begin{array}{c}62,5 \\
(28,01)\end{array}$ & $\begin{array}{c}62,08 \\
(22,809)\end{array}$ \\
\hline 10 semanas & $\begin{array}{c}50 \\
(23,45)\end{array}$ & $\begin{array}{c}55,83 \\
(23,11)\end{array}$ & $\begin{array}{c}52,5 \\
(21,85)\end{array}$ & $\begin{array}{c}55 \\
(18,43)\end{array}$ & $\begin{array}{c}47,5 \\
(21,15)\end{array}$ & $\begin{array}{c}50,83 \\
(20,59)\end{array}$ & $\begin{array}{c}52,5 \\
(16,35)\end{array}$ & $\begin{array}{c}65 \\
(24,49)\end{array}$ & $\begin{array}{c}67,5 \\
(17,54)\end{array}$ \\
\hline
\end{tabular}

Se muestran los umbrales promedio (con desviación estándar) al inicio, 3 semanas y 10 semanas posintervención.

los umbrales promedio de hasta $5,4 \mathrm{~dB}$ entre el control inicial y diez semanas posintervención. No se encontraron diferencias estadísticamente significativas entre los umbrales promedio iniciales, a las tres y diez semanas posintervención en ninguna frecuencia estudiada (Tabla 1, Figura 1).

El análisis de las medianas de los promedios tonales puros (PTP) mostró un aumento de $5 \mathrm{~dB}$ entre el control inicial y tres semanas posteriores a la intervención. Asimismo, se observó una reducción entre las medianas de PTP de 1,3 dB entre el control inicial y el control de las diez semanas. No se encontraron diferencias estadísticamente significativas entre las medianas de PTP de los diferentes controles (Figura 2A). No se observaron variaciones entre las medianas de discriminación entre los parámetros medidos en forma inicial y los controles consecutivos (Figura 2B).

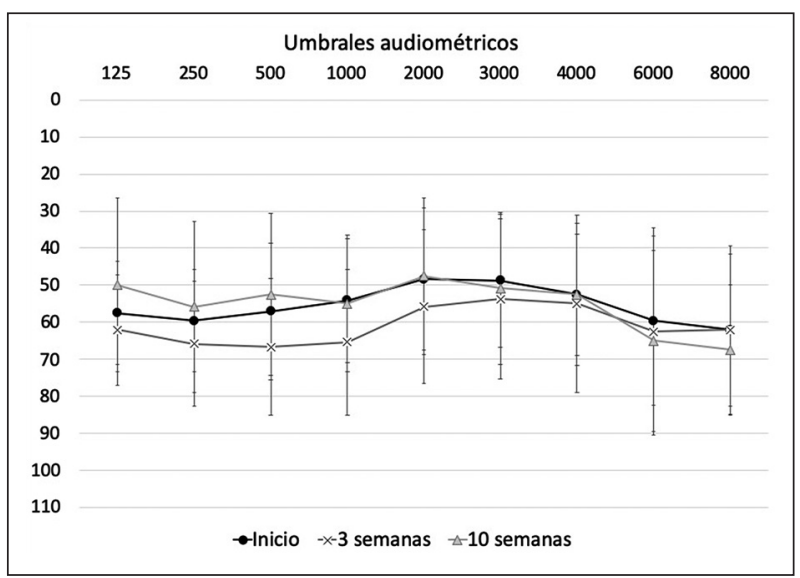

Figura 1. Evolución del promedio de umbrales audiométricos según frecuencia. Se muestra la evolución en el tiempo a las 3 y 10 semanas posintervención de los umbrales promedio (con desviación estándar) para cada frecuencia evaluada. En relación a los umbrales iniciales, se observó un pequeño aumento en los umbrales promedio a las 3 semanas y una reducción de éstos a las 10 semanas (no estadísticamente significativo).

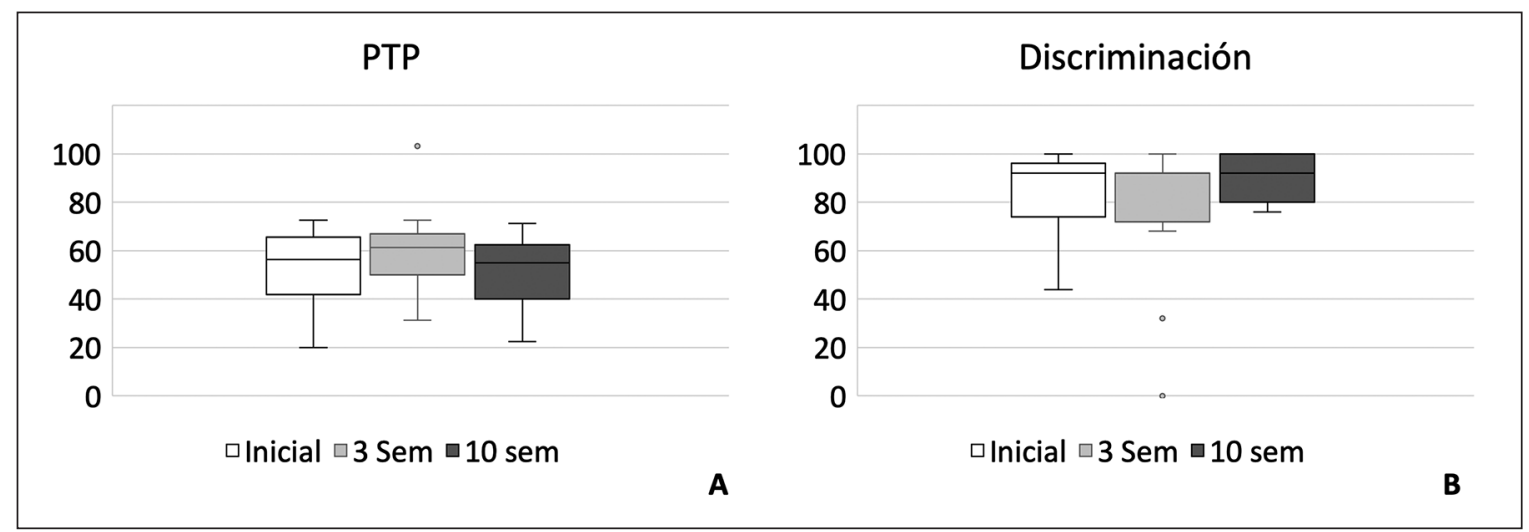

Figura 2. Evolución mediana de PTP y discriminación de la palabra. A: Se muestra mediana de PTP, rango y cuartiles primero y tercero según seguimiento. No se encontraron diferencias estadísticamente significativas entre las medianas de PTP de los diferentes controles. B: Se muestra mediana discriminación, rango y cuartiles primero y tercero según seguimiento. No se observaron variaciones entre las medianas de discriminación entre los parámetros medidos en forma inicial y los controles consecutivos. 


\section{Prueba calórica}

De los doce pacientes evaluados, once $(91,6 \%)$ presentaron algún grado de hipoexcitabilidad por VCL en el oído afectado al inicio del estudio, y un paciente no logró finalizar la evaluación inicial por vértigo intenso. Además, en la evaluación inicial, cinco pacientes $(41,67 \%)$ presentaron hipoexcitabilidad vestibular mayor a $50 \%$ en el oído afectado. Por otra parte, dos pacientes (17\%) presentaron inicialmente vestibulopatía bilateral.

A las tres semanas posintervención, todos los pacientes presentaron hipoexcitabilidad vestibular de algún grado, de los cuales siete $(58 \%)$ presentaron una magnitud mayor a $50 \%$ en el oído afectado. Además, se observó que tres pacientes $(25 \%)$ presentaron vestibulopatía bilateral en este periodo de seguimiento. Finalmente, los siete pacientes que se presentaron al control de las diez semanas posintervención presentaron hipoexcitabili-

\section{Tabla 2. Seguimiento con prueba calórica}

\begin{tabular}{lcc}
\hline & $\begin{array}{c}\text { Hipoexcitabilidad } \\
\mathbf{>} \mathbf{5 0 \%}\end{array}$ & $\begin{array}{c}\text { Vestibulopatía } \\
\text { bilateral }\end{array}$ \\
Inicial & $41,67 \%(5 / 12)$ & $16,67 \%(2 / 12)$ \\
3 semanas & $58,33 \%(7 / 12)$ & $16,67 \%(2 / 12)$ \\
10 semanas & $71,43 \%(5 / 7)$ & $14,29 \%(1 / 7)$ \\
\hline
\end{tabular}

Se muestra el porcentaje de pacientes ( $n$ ) con hipoexcitabilidad vestibular mayor a $50 \%$, y vestibulopatía bilateral al inicio, 3 y 10 semanas posintervención. dad vestibular de algún grado. De ellos, cinco (71\%) presentaron hipoexcitabilidad mayor a $50 \%$ en el oído afectado. Además, un sujeto (14\%) presentó vestibulopatía bilateral (Tabla 2).

\section{Encuesta UCLA-DQ}

El puntaje promedio en la evaluación inicial fue de 20,92 puntos (DE 3,23), con una severidad inicial promedio de las crisis de 17,5 puntos (DE 6,8). A las tres semanas de seguimiento, se observó una disminución estadísticamente significativa del puntaje promedio hasta 15,27 puntos (DE 5,31; $p=0,005)$, y del puntaje de promedio de severidad hasta 6,4 puntos en comparación con el puntaje inicial (DE 4,8; $p=0,005)$. Asimismo, también se observó una disminución estadísticamente significativa a las diez semanas, y a los seis meses posintervención en comparación con la evaluación inicial, presentando puntajes promedio totales de 12,2 puntos (DE 5,4; $p=0,043$ ) y 15,09 (DE $5,14 ; p=0,004)$, respectivamente. Además, la severidad también presentó diferencias estadísticamente significativas a las diez semanas y seis meses posintervención en contraste con la evaluación inicial con índices promedio de 4,6 puntos (DE 4,$6 ; p=0,043$ ) y 7,9 (DE 5,3 ; $p=0,003)$ respectivamente (Tabla 3 , Figura 3 ).

\section{Encuesta DHI}

El puntaje promedio de la evaluación inicial en relación a la encuesta de discapacidad vestibular fue de 44,67 puntos (DE 15,26). No se observaron variaciones estadísticamente

\section{Tabla 3. Puntajes promedio en encuesta UCLA}

\begin{tabular}{|c|c|c|c|c|c|c|c|c|c|c|}
\hline & $n$ & I & II & III & IV & V & Total & $P$ & Severidad & $P$ \\
\hline Inicial & 12 & $\begin{array}{c}3,83 \\
(1,19)\end{array}$ & $\begin{array}{c}4,17 \\
(1,26)\end{array}$ & $\begin{array}{c}4,42 \\
(0,79)\end{array}$ & $\begin{array}{c}3,62 \\
(1,16)\end{array}$ & $\begin{array}{c}3,92 \\
(0,87)\end{array}$ & $\begin{array}{l}20,92 \\
(3,23)\end{array}$ & & $\begin{array}{l}17,5 \\
(6,8)\end{array}$ & \\
\hline 3 semanas & 12 & $\begin{array}{c}2,18 \\
(1,32)\end{array}$ & $\begin{array}{c}2,62 \\
(1,27)\end{array}$ & $\begin{array}{c}3,33 \\
(1,23)\end{array}$ & $\begin{array}{c}3,27 \\
(1,19)\end{array}$ & $\begin{array}{c}3,23 \\
(1,53)\end{array}$ & $\begin{array}{r}15,17 \\
(5,31)\end{array}$ & 0,005 & $\begin{array}{c}6,4 \\
(4,8)\end{array}$ & 0,005 \\
\hline 10 semanas & 5 & $\begin{array}{c}1,8 \\
(1,3)\end{array}$ & $\begin{array}{c}2,2 \\
(1,1)\end{array}$ & $\begin{array}{c}2,4 \\
(0,89)\end{array}$ & $\begin{array}{c}2,6 \\
(1,51)\end{array}$ & $\begin{array}{c}2,75 \\
(0,96)\end{array}$ & $\begin{array}{r}12,2 \\
(5,4)\end{array}$ & 0,043 & $\begin{array}{c}4,6 \\
(4,6)\end{array}$ & 0,043 \\
\hline 6 meses & 11 & $\begin{array}{c}2,18 \\
(1,17)\end{array}$ & $\begin{array}{c}3,08 \\
(1,62)\end{array}$ & $\begin{array}{c}2,75 \\
(1,55)\end{array}$ & $\begin{array}{c}3,18 \\
(1,40)\end{array}$ & $\begin{array}{l}3,36 \\
(1,2)\end{array}$ & $\begin{array}{l}15,09 \\
(5,14)\end{array}$ & 0,004 & $\begin{array}{c}7,9 \\
(5,3)\end{array}$ & 0,003 \\
\hline
\end{tabular}

Se muestra el puntaje promedio (con desviación estándar) para cada ítem de la encuesta. Además, se muestra el total (suma de los ítems) y severidad (se obtiene al multiplicar los ítems I y II). Se expresa el valor $P$ del test rangos signados de Wilcoxon para evaluar diferencias en relación al puntaje inicial y severidad inicial. 
significativas al comparar el promedio inicial con los controles posteriores, los cuales mostraron puntajes promedio de 45,5 (DE 12,87; $p=0,64)$ a las tres semanas, 43,2 (DE 17,15; $p=0,49)$ a las diez semanas y 45,82 (DE 15,46; $p=0,48$ ) seis meses posintervención (Tabla 4 , Figura 4).

Asimismo, no se observaron diferencias estadísticamente significativas al comparar los promedios iniciales de cada uno de los 3 aspectos que componen la encuesta (emocional, funcional y físico) con los controles posteriores. El componente emocional mostró un puntaje promedio inicial de 16,3 (DE 6), 16,4 $(\mathrm{DE} 5 ; p=0,69)$ a las tres semanas, $14,8(\mathrm{DE}$ $6 ; p=0,68)$ a las diez semanas y 16,7 (DE 5,6; $\mathrm{p}=0,51)$ a los seis meses. El aspecto funcional mostró un puntaje promedio inicial de 16 (DE $5,5), 15,9(\mathrm{DE} 5,7 ; p=0,80)$ a las tres semanas, $15,6(\mathrm{DE} 7 ; p=1,0)$ a las diez semanas y 16,7 $(\mathrm{DE} 6,7 ; p=0,44)$ a los seis meses. Por último, el aspecto físico mostro un puntaje promedio inicial de 12,3 (DE 4,3), 13,1 (DE 4; $p=0,47)$ a las tres semanas, $12,8(\mathrm{DE} 5,5 ; p=0,5)$ a las diez semanas y $12,4(\mathrm{DE} 4,8 ; p=0,76)$ a los seis meses.

\section{Entrevista a los seis meses}

A los seis meses de haber recibido las inyecciones, $66,7 \%$ de los pacientes (8/12) refirió haber tenido al menos 1 crisis de vértigo en los últimos seis meses. De ellos, el 75\% (6/8) caracterizó estas crisis como de menor intensidad, y $25 \%$ (2/8) de igual intensidad. Ninguno de los sujetos reportó crisis de mayor intensidad. Además, el 88\% (7/8) de aquellos sujetos que presentaron crisis, reportaron una menor duración de éstas, y 1 de ellos de igual duración. Ningún sujeto reportó crisis de mayor duración. El 91,6\% (11/12) de los sujetos volvería a colocarse las inyecciones de corticoides transtimpánicos para su patología de base. Un sujeto reportó que no volvería a usarlos dado su costo elevado. Finalmente, el $75 \%$ de los sujetos (9/12) refirieron menor ansiedad frente a la posibilidad de una nueva crisis.

\section{Discusión}

La enfermedad de Ménière es una entidad clínica compleja con una gran variedad de tra-

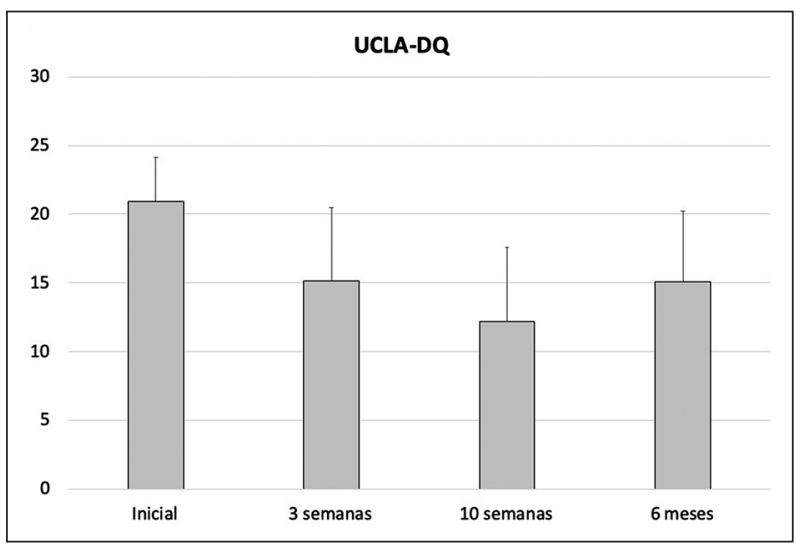

Figura 3. Puntajes promedios en la encuesta UCLA-DQ. Se muestran los puntajes promedios (con desviación estándar) en la encuesta UCLA-DQ al inicio, 3 semanas, 10 semanas y 6 meses posintervención. Se observó una disminución estadísticamente significativa del puntaje promedio en esta encuesta en relación con el puntaje inicial a las 3 semanas que se mantuvo hasta los 6 meses inclusive.

Tabla 4. Puntajes promedio en encuesta DHI

\begin{tabular}{lccc}
\hline & $\mathbf{n}$ & Promedio & $\boldsymbol{p}$ \\
Inicial & 12 & $44,67(15,27)$ & \\
3 semanas & 12 & $45,5(12,87)$ & 0,64 \\
10 semanas & 5 & $43,2(17,15)$ & 0,49 \\
6 meses & 11 & $45,82(15,46)$ & 0,48 \\
\hline $\begin{array}{l}\text { Se muestra el puntaje promedio (con desviación estándar). Se } \\
\text { expresa el valor } P \text { del test rangos signados de Wilcoxon para } \\
\text { evaluar diferencias en relación al puntaje inicial. }\end{array}$ \\
\hline
\end{tabular}

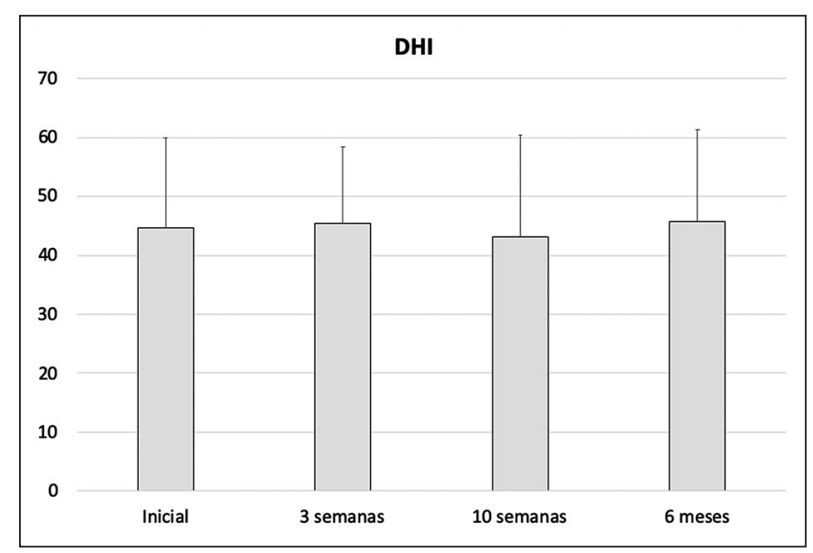

Figura 4. Puntajes promedios de la encuesta DHI. Se muestran los puntajes promedios (con desviación estándar) en la encuesta DHI al inicio, 3 semanas, 10 semanas y 6 meses posintervención. No se observaron variaciones estadísticamente significativas al comparar el promedio inicial con los controles posteriores. 
tamientos, cuyo objetivo es intentar prevenir o disminuir la intensidad del vértigo y retrasar la progresión de la pérdida auditiva ${ }^{12,20}$. Dentro de los tratamientos invasivos, el uso de corticoides transtimpánicos es considerada una alternativa terapéutica no ablativa que minimiza los riesgos atribuibles al uso de corticoides sistémicos, y que puede ser considerada ante una falla al tratamiento médico ${ }^{5,12,21}$. El presente estudio expone la experiencia de nuestro centro en relación al manejo de pacientes con enfermedad de Ménière de difícil tratamiento utilizando esta alternativa terapéutica ocupando $4 \mathrm{mg} / \mathrm{ml}$ de dexametasona, y colocando tres inyecciones en 10 días.

Desde el punto de vista epidemiológico, el perfil demográfico de los pacientes en nuestro estudio es similar al de publicaciones internacionales. Se observó que la mediana de edad fue de 54 años, lo cual es concordante con los promedios de edad de inicio de la enfermedad, que pueden variar entre los 40-60 años ${ }^{1,22}$. Por otro lado, se obtuvo una relación de 1:1 entre mujeres y hombres, lo cual difiere de lo reportado en la literatura en que se reporta una mayor frecuencia en población femenina $(1,89-4,3: 1)^{1,12,22,23}$. Esto puede deberse al número limitado de participantes en nuestro estudio, sin embargo, otras publicaciones con poblaciones comparables y tamaños muestrales similares han reportado incluso una mayor proporción de hombres ${ }^{21,24}$. Lo anterior podría implicar que los pacientes con enfermedad de Ménière de difícil manejo podrían tener un perfil demográfico distinto al que se reporta generalmente. No obstante, se requieren estudios con tamaños muestrales mayores para apoyar esta afirmación.

En relación a las evaluaciones audiométricas, se observaron variaciones de los umbrales de ciertas frecuencias entre el control inicial y el de las tres semanas posintervención de hasta 11,25 dB (125-3.000 Hz). Sin embargo, estos cambios no fueron constantes en el tiempo, ya que los controles subsecuentes mostraron fluctuaciones de los umbrales entre 5,4 y 7,5 dB en algunos rangos frecuenciales (3.000-8.000 $\mathrm{Hz}$ y $125-2.000 \mathrm{~Hz}$ respectivamente). Estos cambios pueden ser explicados por la variabilidad inter-evaluador del personal de salud que realiza el examen, que puede dar cuenta entre 5-10 dB de diferencia, o como parte de la evolución de la enfermedad ya que por definición los síntomas de ésta fluctúan ${ }^{5,12,15,25}$. Además, al evaluar las medianas de los PTP, también se observaron variaciones de $5 \mathrm{~dB}$ entre el primer control y el de las tres semanas, y de 1,3 dB entre el primer control y el de las diez semanas. Estas diferencias no concuerdan con los resultados de Molnár y cols. ${ }^{26}$, donde se reportan mejorías de PTP entre 15 a $40 \mathrm{~dB}$, sin embargo, otros estudios no han mostrado diferencias estadísticamente significativas en la variación de los PTP posterior, la utilización de corticoides transtimpánicos ${ }^{26-29}$. Las variaciones en los PTP observadas en nuestro estudio no fueron clínica ni estadísticamente significativas, y también pueden ser explicadas por diferencias inter-evaluador.

Respecto a los resultados de las evaluaciones con prueba calórica, se observó que un 91,6\% de los pacientes evaluados presentaron algún grado de hipoexcitabilidad en el oído afectado. Esto supera los valores expuestos en múltiples estudios donde se reporta que hasta un $65 \%$ de los pacientes con enfermedad de Ménière puede presentar hipoexcitabilidad unilateral en la prueba calórica ${ }^{12,30-32}$. El elevado porcentaje de alteraciones obtenido en nuestro estudio podría explicarse por el perfil de los pacientes evaluados, ya que todos ellos presentan una enfermedad de larga data con un tiempo de evolución media de 9,5 años (rango 2,3-16 años) y un mal control de las crisis. Sin embargo, Huppert y cols. ${ }^{33}$ plantean que los resultados de la prueba calórica pueden ser impredecibles en el tiempo, por lo que las alteraciones de este examen durante la evolución de esta patología pueden ser variables $^{33}$. Además, la prueba calórica resulta ser particularmente útil en la evaluación de los pacientes con enfermedad de Ménière, ya que existe un aumento significativo del contenido del conducto membranoso, lo cual, alteraría el mecanismo hidrostático ${ }^{34}$.

Adicionalmente, se observó que un $41,67 \%$ de los pacientes presentaron en forma inicial una hipoexcitabilidad vestibular mayor a 50\% en el oído afectado. Si bien esto podría asociarse a cuadros de mayor intensidad o de evolución prolongada, se ha reportado que los resultados de exámenes vestibulares, como la prueba calórica, se presentan una baja correlación con el grado de daño y la per- 
cepción de discapacidad del paciente ${ }^{12,35}$. Los resultados de la prueba calórica presentaron variaciones entre el control inicial, a las tres y diez semanas posintervención, no obstante, estas diferencias no fueron estadísticamente significativas.

Por otro lado, la utilización de las encuestas buscó indagar en dos aspectos importantes en esta patología: el impacto del vértigo en el individuo (UCLA-DQ) y el grado de discapacidad percibida por el paciente producto del vértigo (DHI). Los resultados de la encuesta UCLA-DQ mostraron una mejoría significativa en los puntajes obtenidos en los controles posintervención en relación a la evaluación inicial, observando una disminución de al menos cinco puntos, la cual fue sostenida durante los seis meses de seguimiento. Además, se observó una disminución 10 puntos en el puntaje de severidad calculado que se mantuvo constante durante el seguimiento. Estos resultados son compatibles con los publicados por Patel y cols. ${ }^{36}$ donde se observó que, en todos los estudios revisados, algún porcentaje de los pacientes mostró mejoría del vértigo y, por ende, reducción en el impacto que esta patología produce en cada individuo ${ }^{36}$. En contraste, no se encontraron diferencias estadísticamente significativas en ninguno de los controles utilizando la encuesta DHI como puntaje global ni en el análisis por subcomponentes (emocional, funcional ni físico). Estos resultados no son concordantes con los reportados por Pradhan y cols. ${ }^{27}$ donde se observaron reducciones de hasta 40 puntos en distintas etapas de seguimiento ${ }^{27}$. Esta discordancia puede explicarse por la diferencia en los valores iniciales obtenidos en la DHI, ya que en el estudio antes mencionado el puntaje inicial promedio fue de 91,58 puntos y en nuestro estudio el promedio inicial fue de 44,67. Tomando en consideración que los pacientes del estudio de Pradhan y cols. presentaban una percepción de discapacidad mayor que los pacientes en nuestro estudio, podría ser esperable que la disminución en los puntajes fuera mayor en ese grupo. Por otra parte, siempre hay que considerar que el efecto placebo puede jugar un rol importante al momento de analizar e interpretar los resultados de encuestas de percepción. Esto puede explicarse ya que, dada la naturaleza fluctuante de esta patología, el uso de corticoides intratim- pánicos (CIT) puede coincidir con momentos de remisión secundarios a la evolución natural de la enfermedad. Sin embargo, tampoco es descartable que los CIT tengan algún rol en el control sintomático de la enfermedad de Ménière con los datos obtenidos.

Finalmente, en relación con los resultados de la encuesta de los seis meses, se observó que un $75 \%$ de los pacientes presentaron crisis de menor intensidad y un $88 \%$ reportaron que fueron de menor duración. Además, casi la totalidad de los pacientes intervenidos refirieron que volverían a utilizar corticoides transtimpánicos para el manejo de su patología. Estas percepciones son compatibles con los resultados obtenidos en la encuesta UCLADQ, lo cual nos lleva a pensar que la presente intervención podría mejorar el impacto que tiene la enfermedad en los pacientes. Esto puede fundamentarse en la gran correlación que tiene este instrumento y el amplio uso que se le ha dado en la literatura para evaluar el impacto que tiene esta patología en la vida de los pacientes ${ }^{37}$. Nuestro estudio es descriptivo y representa nuestra experiencia local. Se requieren estudios clínicos randomizados, o caso-control, para poder definir qué corticoide, y qué protocolo, darán los mejores resultados clínicos.

\section{Conclusión}

Si bien la evidencia del uso de corticoides transtimpánicos como tratamiento de la enfermedad de Ménière de difícil manejo aún es controvertida, los resultados del presente estudio indican que el uso de dexametasona transtimpánica puede ayudar a disminuir el impacto que tiene esta patología en la vida de los pacientes.

\section{Bibliografía}

1. Nakashima T, Pyykkö I, Arroll MA, et al. Meniere’s disease. Nat Rev Dis Prim. 2016;2:1-19. doi: 10.1038/ nrdp.2016.28.

2. Harcourt J, Barraclough K, Bronstein AM. Meniere's disease. BMJ. 2014;349:g6544-g6544. doi: 10.1136/ bmj.g6544.

3. Espinosa-Sánchez JM, López-Escamez JA. Menière’s disease. In: Handbook of Clinical Neurology. Vol 137. 
1st ed. Elsevier B.V.; 2016:257-277. doi:10.1016/B9780-444-63437-5.00019-4.

4. Magnan J, Ozgirgin ON, Trabalzini F, et al. European Position Statement on Diagnosis, and Treatment of Meniere's Disease. J Int Adv Otol. 2018;14(2):317-321. doi: 10.5152/iao.2018.140818.

5. Nevoux J, Barbara M, Dornhoffer J, Gibson W, Kitahara T, Darrouzet V. International consensus (ICON) on treatment of Ménière's disease. Eur Ann Otorhinolaryngol Head Neck Dis. 2018;135(1):S29-S32. doi: 10.1016/j. anorl.2017.12.006.

6. Crowson MG, Patki A, Tucci DL. A Systematic Review of Diuretics in the Medical Management of Ménière's Disease. Otolaryngol - Head Neck Surg (United States). 2016;154(5):824-834. doi: 10.1177/0194599816630733.

7. Hussain K, Murdin L, Schilder AGM. Restriction of salt, caffeine and alcohol intake for the treatment of Ménière's disease or syndrome. Cochrane Database Syst Rev. 2018;2018(12). doi: 10.1002/14651858. CD012173.pub2.

8. Claes J. A Review of Medical Treatment for Ménière's Disease. Acta Otolaryngol. 2000;120(544):34-39. doi: 10.1080/000164800750044461.

9. Syed MI, Ilan O, Nassar J, Rutka JA. Intratympanic therapy in Meniere's syndrome or disease: Up to date evidence for clinical practice. Clin Otolaryngol. 2015;40(6):682-690. doi: 10.1111/coa.12449.

10. Phillips JS, Westerberg B. Intratympanic steroids for Ménière's disease or syndrome. Cochrane Database Syst Rev. 2011;(7). doi: 10.1002/14651858.cd008514. pub2.

11. Stachler RJ, Chandrasekhar SS, Archer SM, et al. Clinical practice guideline: Sudden hearing loss. Otolaryngol - Head Neck Surg. 2012;146(S3). doi: 10.1177/0194599812436449.

12. Basura GJ, Adams ME, Monfared A, et al. Clinical Practice Guideline: Ménière's Disease. Otolaryngol - Head Neck Surg (United States). 2020;162:S1-S55. doi: 10.1177/0194599820909438.

13. Derebery MJ, Rao VS, Siglock TJ, Linthicum FH, Nelson RA. Meniere's Disease: An Immune ComplexMediated Illness? Laryngoscope. 1991;101(3):225-229. doi: 10.1288/00005537-199103000-00001.

14. Committee on Hearing and Equilibrium guidelines for the diagnosis and evaluation of therapy in Menière's disease. American Academy of Otolaryngology-Head and Neck Foundation, Inc. Otolaryngol Head Neck Surg. 1995;113(3):181-185. doi: 10.1016/S0194-5998(95)70102-8.

15. López-Escamez JA, Carey J, Chung WH, et al. Criterios diagnósticos de enfermedad de Menière: Documento de consenso de la Bárány Society, la Japan Society for Equilibrium Research, la European Academy of Otology and Neurotology (EAONO), la American Academy of Otolaryngology-Head and Neck Surgery (AA). Acta Otorrinolaringol Esp. 2016;67(1):1-7. doi: 10.1016/j.otorri.2015.05.005.

16. Pérez-Vázquez P, Franco-Gutiérrez V. La prueba calórica. Rev ORL. 2018:22. doi: 10.14201/orl.17699.

17. Strupp M, Kim JS, Murofushi T, et al. Bilateral vestibulopathy: Diagnostic criteria consensus document of the classification committee of the barany society. J Vestib Res Equilib Orientat. 2017;27(4):177-189. doi: 10.3233/VES-170619.

18. Peña A. Homologación lingüística nacional del Dizziness Handicap Inventory (test de discapacidad vestibular). Rev Otorrinolaringol Cir Cabeza Cuello. 2011;71(1):85-88. doi: 10.4067/S071848162011000100013.

19. Novoa I, Donoso S, Martínez Y, Mercado A, Pino C, Mercado V. Efectividad de cinco sesiones de rehabilitación vestibular en mujeres mayores de 60 años con hipofunción vestibular. Rev Otorrinolaringol Cir Cabeza Cuello. 2018;78(3):259-266. doi: 10.4067/ s0717-75262018000300259.

20. Naples JG, Henry L, Brant JA, Eliades SJ, Ruckenstein MJ. Intratympanic Therapies in Ménière Disease: Evaluation of Outcomes and Early Vertigo Control. Laryngoscope. 2019;129(1):216-221. doi: 10.1002/ lary.27392.

21. Jumaily M, Faraji F, Mikulec AA. Intratympanic Triamcinolone and Dexamethasone in the Treatment of Ménières Syndrome. Otol Neurotol. 2017;38(3):386-391. doi: 10.1097/ MAO.0000000000001311.

22. Alexander TH, Harris JP. Current Epidemiology of Meniere's Syndrome. Otolaryngol Clin North Am. 2010;43(5):965-970. doi: 10.1016/j.otc.2010.05.001.

23. López-Escamez JA, Carey J, Chung WH, et al. Diagnostic criteria for Menière's disease. J Vestib Res Equilib Orientat. 2015;25(1):1-7. doi: 10.3233/VES150549.

24. Paragache G, Panda NK, Ragunathan M, Sridhara. Intratympanic dexamethasone application in Meniere's disease - Is it superior to conventional therapy? Indian J Otolaryngol Head Neck Surg. 2005;57(1):21-23. doi: 10.1007/BF02907620.

25. Katz J, Chasin M, English K, Hood LJ, Tillery KL. Handbook of Clinical Audiology: Seventh Edition. 2014.

26. Molnár A, Maihoub S, Tamás L, Szirmai Á. Intratympanically administered steroid for progressive sensorineural hearing loss in Ménière's disease. Acta Otolaryngol. 2019;139(11):982-986. doi: 10.1080/00016489.2019.1658898.

27. Pradhan P, Lal P, Sen K. Long Term Outcomes of Intratympanic Dexamethasone in Intractable Unilateral Meniere's Disease. Indian J Otolaryngol Head Neck Surg. 2019;71:1369-1373. doi: 10.1007/ s12070-018-1431-3.

28. Schoo DP, Tan GX, Ehrenburg MR, Pross SE, Ward BK, Carey JP. Intratympanic (IT) Therapies for Menière's Disease: Some Consensus Among the 
Confusion. Curr Otorhinolaryngol Rep. 2017;5(2):132141. doi: 10.1007/s40136-017-0153-5.

29. Silverstein H, Isaacson JE, Olds MJ, Rowan PT, Rosenberg S. Dexamethasone inner ear perfusion for the treatment of Meniere's disease: a prospective, randomized, double-blind, crossover trial. Am J Otol. 1998;19(2):196-201. doi: 10.1111/j.1467-6494.1954. tb02338.x.

30. Cordero-Yanza JA, Arrieta Vázquez EV, Hernaiz Leonardo JC, Mancera Sánchez J, Hernández Palestina MS, Pérez-Fernández N. Comparative study between the caloric vestibular and the video-head impulse tests in unilateral Menière's disease. Acta Otolaryngol. 2017;137(11):1178-1182. doi: 10.1080/00016489.2017.1354395.

31. Lee SU, Kim HJ, Koo JW, Kim JS. Comparison of caloric and head-impulse tests during the attacks of Meniere's disease. Laryngoscope. 2017;127(3):702-708. doi: 10.1002/lary.26103.

32. Shin JE, Kim CH, Park HJ. Vestibular abnormality in patients with Meniere's disease and migrainous vertigo. Acta Otolaryngol. 2013;133(2):154-158. doi: 10.3109/00016489.2012.727469.

33. Huppert D, Strupp M, Brandt T. Longterm course of Menire's disease revisited. Acta Otolaryngol. 2010;130(6):644-651. doi: $10.3109 / 00016480903382808$.

34. McGarvie LA, Curthoys IS, MacDougall HG, Halmagyi GM. What does the dissociation between the results of video head impulse versus caloric testing reveal about the vestibular dysfunction in Ménière's disease? Acta Otolaryngol. 2015;135(9):859-865. doi: 10.3109/00016489.2015.1015606.

35. Adams ME, Heidenreich KD, Kileny PR. Audiovestibular Testing in Patients with Meniere's Disease. Otolaryngol Clin North Am. 2010;43(5):9951009. doi: 10.1016/j.otc.2010.05.008.

36. Patel M. Intratympanic corticosteroids in Ménière's disease: A mini-review. J Otol. 2017;12(3):117-124. doi: 10.1016/j.joto.2017.06.002.

37. Zur O, Carmeli E. The University of California Los Angeles Dizziness Questionnaire: Advantages and disadvantages. J Vestib Res Equilib Orientat. 2013;23(6):279-283. doi: 10.3233/VES-130480. 